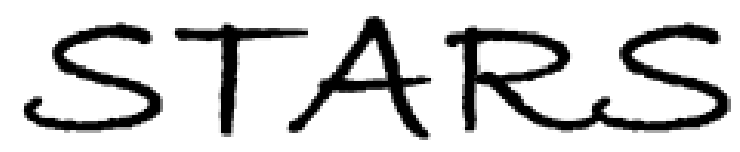

University of Central Florida

STARS

$1-1-2006$

\title{
Twist angle effects on the dynamic response of in-plane- switching liquid crystal displays
}

Yubao Sun

Hongmei Ma

Zhidong Zhang

Xinyu Zhu

University of Central Florida

Shin-Tson Wu

University of Central Florida

Find similar works at: https://stars.library.ucf.edu/facultybib2000 University of Central Florida Libraries http://library.ucf.edu

This Article is brought to you for free and open access by the Faculty Bibliography at STARS. It has been accepted for inclusion in Faculty Bibliography 2000 s by an authorized administrator of STARS. For more information, please contact STARS@ucf.edu.

\section{Recommended Citation}

Sun, Yubao; Ma, Hongmei; Zhang, Zhidong; Zhu, Xinyu; and Wu, Shin-Tson, "Twist angle effects on the dynamic response of in-plane-switching liquid crystal displays" (2006). Faculty Bibliography 2000 s. 6627. https://stars.library.ucf.edu/facultybib2000/6627

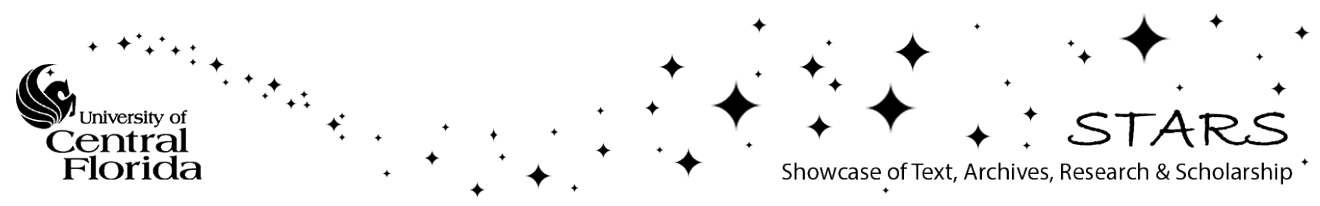




\section{Twist angle effects on the dynamic response of in-plane-switching liquid crystal displays}

Cite as: Appl. Phys. Lett. 89, 041110 (2006); https://doi.org/10.1063/1.2236215

Submitted: 10 February 2006 . Accepted: 03 June 2006 . Published Online: 25 July 2006

Yubao Sun, Hongmei Ma, Zhidong Zhang, Xinyu Zhu, and Shin-Tson Wu

\section{ARTICLES YOU MAY BE INTERESTED IN}

Optimal rubbing angle for reflective in-plane-switching liquid crystal displays

Applied Physics Letters 81, 4907 (2002); https://doi.org/10.1063/1.1532534

Electro-optical characteristics and switching behavior of a twisted nematic liquid crystal device based upon in-plane switching

Applied Physics Letters 80, 1847 (2002); https://doi.org/10.1063/1.1459764

Electro-optical characteristics and switching behavior of the in-plane switching mode Applied Physics Letters 67, 3895 (1995); https://doi.org/10.1063/1.115309
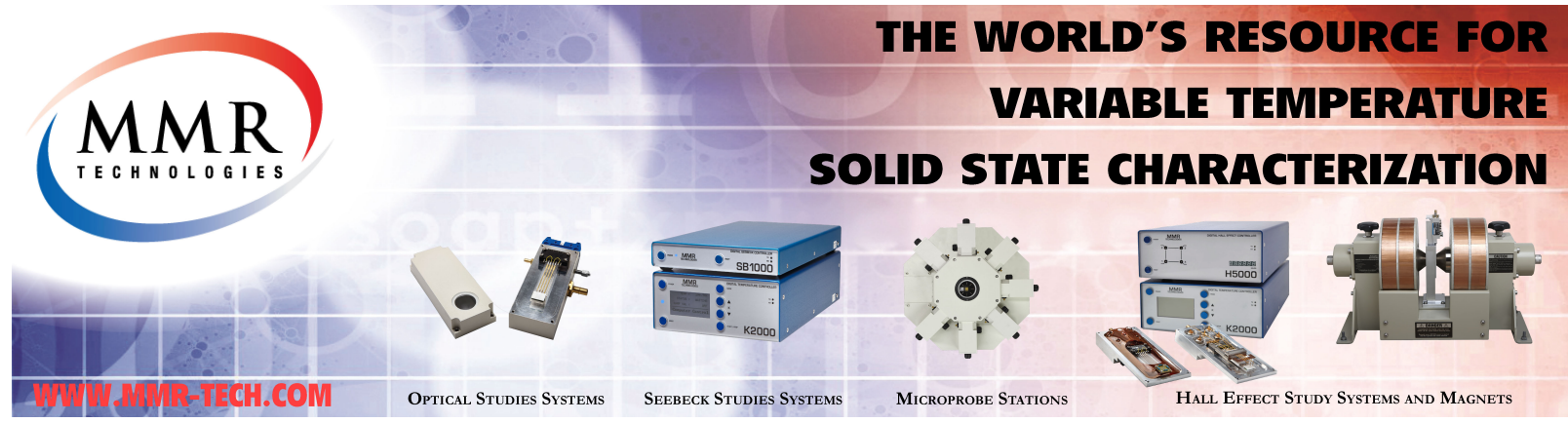


\title{
Twist angle effects on the dynamic response of in-plane-switching liquid crystal displays
}

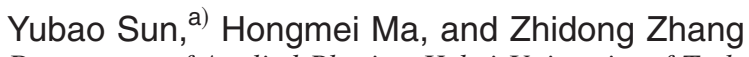 \\ Department of Applied Physics, Hebei University of Technology, Tianjin 300130, \\ People's Republic of China \\ Xinyu Zhu and Shin-Tson Wu \\ College of Optics and Photonics, University of Central Florida, Orlando, Florida 32816
}

(Received 10 February 2006; accepted 3 June 2006; published online 25 July 2006)

\begin{abstract}
Twist angle effects on the response time of in-plane-switching liquid crystal displays are analyzed. The authors propose a device configuration whose top and bottom boundary liquid crystal layers are symmetric to each other with respect to the electric field direction. The analytical results of this device configuration indicate that the response time is improved at least four times faster than that of a conventional in-plane-switching twisted-nematic mode and normal in-plane-switching mode.

(C) 2006 American Institute of Physics. [DOI: 10.1063/1.2236215]
\end{abstract}

In-plane-switching (IPS) mode ${ }^{1-3}$ has been widely used for large-screen transmissive liquid crystal displays (LCDs) because of its wide viewing angle, weak color shift, and high contrast ratio. However, the response time of the IPS mode is relatively slow as compared to that of other display modes because of its smaller twist elastic constant. ${ }^{4}$ In the IPS mode, the effect of rubbing angle on LC director's response time has been analyzed in detail. ${ }^{5-7}$ However, the twist angle effect has not yet been analyzed due to the difficulty in obtaining analytical solutions.

In this letter, we present two in-plane-switching twistednematic (TN) configurations and derive the corresponding LC director's dynamic response by solving the EricksonLeslie equation under small angle approximation. The twist angle effects are analyzed quantitatively in the range of $0^{\circ}-180^{\circ}$.

Figures 1(a) and 1(b) show the director configurations of two different IPS-TN modes under study. In both figures, $\Phi$ represents the total initial twist angle. In Fig. 1(a) the LC director on the bottom substrate is perpendicular to the electric field direction. However, in Fig. 1(b) both top and bottom boundary layers are symmetric with respect to the electric field direction. From Fig. 1(b), we find $\Phi=\pi-2 \alpha$, where $\alpha$ is the initial LC alignment direction (i.e., rubbing angle) with respect to the vertical reference. Figure 1(a) is known as the IPS-TN mode when the total twist angle is $\Phi \leqslant \pi / 2.8,9$ On the other hand, if the twist angle is between $\pi / 2$ and $\pi$, it is called IPS supertwisted nematic (STN) ${ }^{10}$ as Fig. 1(b) depicts.

When the backflow and inertial effects are ignored, the dynamics of LC director rotation is described by the following Erickson-Leslie equation:, ${ }^{41}$

$$
\gamma_{1} \frac{\partial \phi}{\partial t}=K_{22} \frac{\partial^{2} \phi}{\partial z^{2}}+\varepsilon_{0}|\Delta \varepsilon| E^{2} \sin \phi \cos \phi,
$$

where $\gamma_{1}$ is the rotational viscosity, $K_{22}$ is the twist elastic constant, $\Delta \varepsilon$ is the dielectric anisotropy, $E$ is the electric field strength, and $\phi$ is the LC director rotation angle. Here the $z$ axis is along the substrate normal direction and the LC cell

\footnotetext{
${ }^{a)}$ Electronic mail: hmtj450@vip.sina.com
}

gap is $d$. In our analysis, we assume the strong surface anchoring so that the bottom and top boundary layers are fixed even under applied voltage. For the IPS-TN cell shown in Fig. 1(a), the middle layer reaches the maximum twist angle deformation $\phi_{m}$ in the voltage-on state. A similar condition occurs at the quarter and three-quarter layers in the IPS-STN cell. Using these initial conditions, in the steady state we can approximately express the electric field induced twist angle $\phi$ deformation along the $z$ axis as ${ }^{12}$

$$
\phi(z)=\phi_{1}(z)+\phi_{m} \sin (\pi z / d),
$$

and

$$
\phi(z)=\phi_{2}(z)+\phi_{m} \sin (2 \pi z / d),
$$

for IPS-TN and IPS-STN, respectively. Here, $\phi_{1}(z)=\Phi z / d$ and $\phi_{2}(z)=\alpha+\Phi z / d$ are the twist profiles under zero electric field for Figs. 1(a) and 1(b), respectively.

First, let us consider the relaxation process. We assume that the electric field is removed instantaneously at time $t=0$. During the relaxation period from the activated state to the initial state, the LC director deformation can be expressed by the following exponential forms:

$$
\phi(z, t)=\phi_{1}(z)+\phi_{m} \sin (\pi z / d) \exp (-t / \tau),
$$

and

$$
\phi(z, t)=\phi_{2}(z)+\phi_{m} \sin (2 \pi z / d) \exp (-t / \tau),
$$

for IPS-TN and IPS-STN cells, respectively. Substituting Eqs. (3a) and (3b) into Eq. (1), the relaxation time can be solved easily:

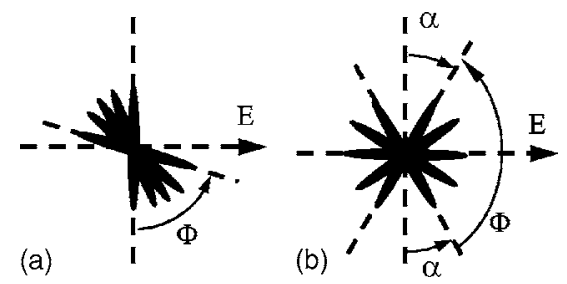

FIG. 1. Two different LC director profiles in the LC cell for (a) conventional IPS-TN mode with LC director on the bottom substrate perpendicular to the electric field and (b) symmetric IPS-STN mode with LC director of the middle layer parallel to the electric field. 


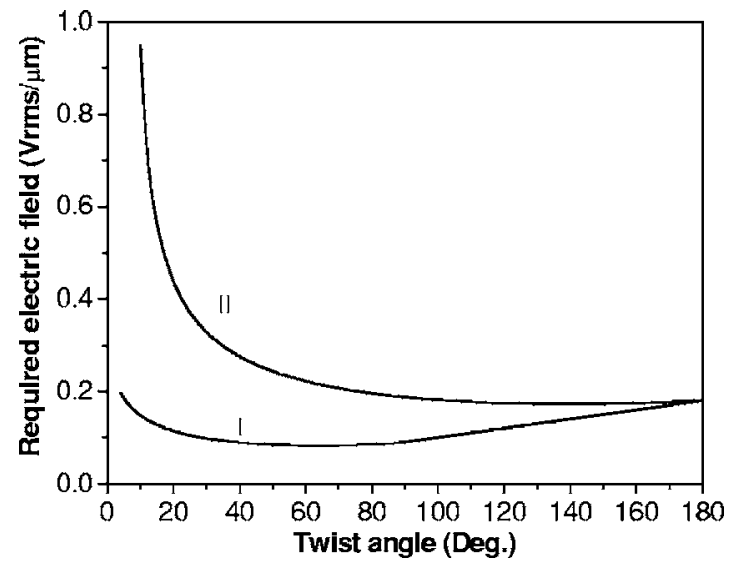

FIG. 2. Required driving electric field for $\phi_{m}=0.05$ in the two configurations with different twist angles. Curve I is for the configuration of Fig. 1(a), and curve II is for the configuration of Fig. 1(b).

$$
\tau_{\text {off }}=\gamma_{1} d^{2} /\left(\pi^{2} K_{22}\right)
$$

for the IPS-TN mode shown in Fig. 1(a), and

$$
\tau_{\text {off }}=\gamma_{1} d^{2} /\left(4 \pi^{2} K_{22}\right),
$$

for the IPS-STN mode shown in Fig. 1(b).

Equations (4a) and (4b) indicate that the relaxation time of both twist configurations is governed by the cell gap $d$ and the LC viscoelastic coefficient $\left(\gamma_{1} / K_{22}\right)$. Clearly, we find that the IPS-STN cell has four times faster response time than the conventional IPS-TN mode. The LC directors at the middle plane of the IPS-STN mode, whose direction is parallel to the electric field direction, remain unchanged at the voltage-on state. Thus the IPS-STN is equivalent to two identical IPS-TN cells, each of which has one-half of the LC layer thickness of the original cell. As compared to the IPS-TN cell, the IPS-STN cell should have four times faster relaxation time provided that both configurations have the same cell gap $d$.

Next, we analyze the rise time. The LC director deformation during the rise period can be described as ${ }^{12}$

$$
\phi(z, t)=\phi_{1}(z)+\phi_{m} \sin (\pi z / d)[1-\exp (-2 t / \tau)],
$$

for the IPS-TN cell, and

$$
\phi(z, t)=\phi_{2}(z)+\phi_{m} \sin (2 \pi z / d)[1-\exp (-2 t / \tau)],
$$

for the IPS-STN cell. If $x_{1}=\phi_{m} \sin (\pi z / d)$ and $x_{2}$ $=\phi_{m} \sin (2 \pi z / d)$, then with the small angle approximation approach we have $x_{i} \ll 1, \cos \left(2 x_{i}\right) \approx 1$, and $\sin \left(2 x_{i}\right) \approx 2 x_{i}$, where $i=1$ and 2. Substituting Eqs. (5a) and (5b) into Eq. (1), we obtain the rise time for the IPS-TN and IPS-STN cells as

$$
\tau_{1}=2 \gamma_{1} /\left\{\exp (2) \varepsilon_{0} \Delta \varepsilon E^{2} X_{1}-\pi^{2} K_{22}[\exp (2)-1] / d^{2}\right\},
$$

and

$$
\tau_{2}=2 \gamma_{1} /\left\{\exp (2) \varepsilon_{0} \Delta \varepsilon E^{2} X_{2}-4 \pi^{2} K_{22}[\exp (2)-1] / d^{2}\right\},
$$

respectively, where $\quad X_{i}=\int_{0}^{d}\left\{\sin \left[2 \phi_{i}(z)\right]+2 x_{i}[1\right.$ $\left.-\exp (2)] \cos \left[2 \phi_{i}(z)\right]\right\} d z / \int_{0}^{d} x_{i} d z, \quad i=1$ and 2. Under small angle approximation, Eqs. (6a) and (6b) can be, respectively, rewritten as
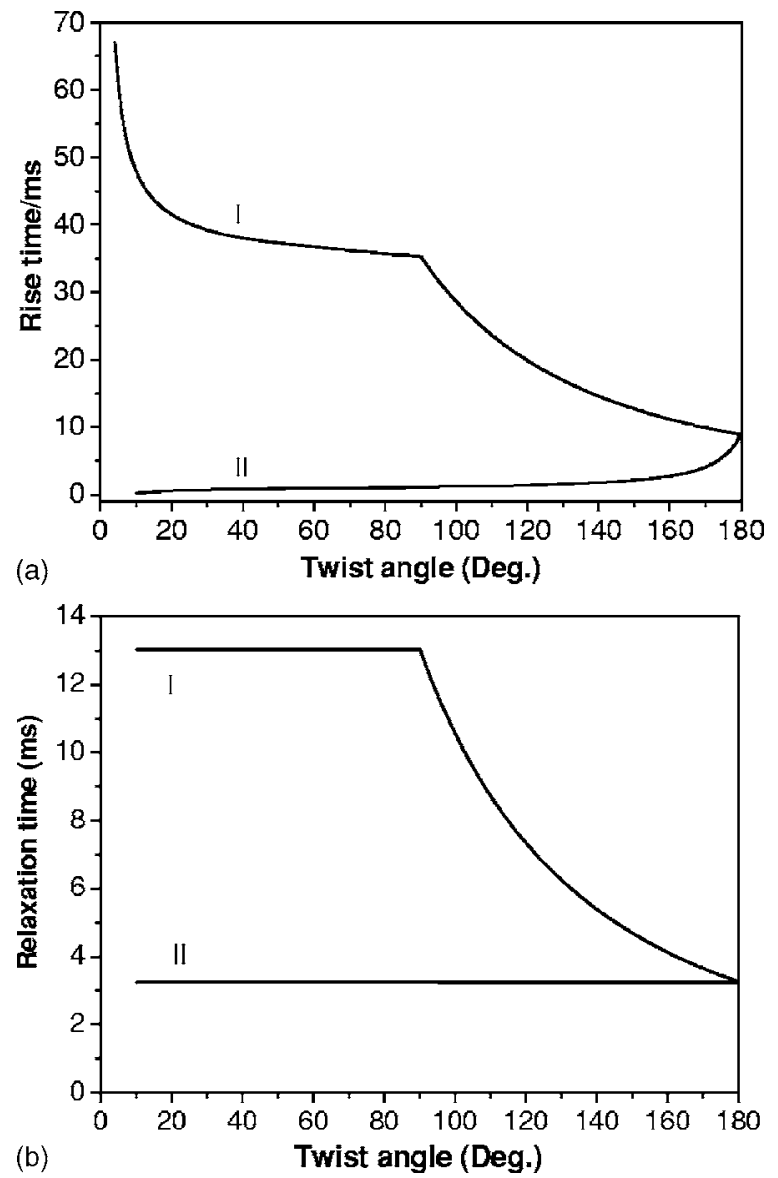

FIG. 3. (a) Rise time and (b) relaxation time for the two configurations with different twist angles. Curve I is for the configuration of Fig. 1(a), and curve II is for the configuration of Fig. 1(b).

$$
\tau_{1}=\gamma_{1} /\left[\varepsilon_{0} \Delta \varepsilon E^{2} /\left(2 \phi_{m 1}\right)-\pi^{2} K_{22} / d^{2}\right]
$$

and

$$
\tau_{2}=\gamma_{1} /\left[\varepsilon_{0} \Delta \varepsilon E^{2} /\left(2 \phi_{m 2}\right)-4 \pi^{2} K_{22} / d^{2}\right]
$$

For a given weak electric field strength, $\phi_{m i}(i=1,2)$ can be obtained from Eq. (1) and has an approximative relation $\phi_{m 1} \approx 4 \phi_{m 2}$ for the two configurations with the same twist angle $(\Phi)$. From Eq. (7), the rise time of IPS-STN configuration is about four times faster than that of IPS-TN configuration while the two configurations are driven by the same low voltage.

Equations (4a), (4b), (6a), and (6b) are the general formulas for calculating the LC director's decay and rise times, assuming the applied voltage is not too high. To compare the switching time for different twist angles, the required electric field to obtain the same maximum twist angle deformation can be calculated from Eq. (1) and expressed as

$$
E_{1}^{2}=\frac{K_{22} \pi^{2}}{d^{2} \varepsilon_{0} \Delta \varepsilon} \frac{\int_{0}^{d} x_{1} d z}{\int_{0}^{d} \sin \left[\phi_{1}(z)+x_{1}\right] \cos \left[\phi_{1}(z)+x_{1}\right] d z},
$$




$$
E_{2}^{2}=\frac{4 K_{22} \pi^{2}}{d^{2} \varepsilon_{0} \Delta \varepsilon} \frac{\int_{0}^{d} x_{2} d z}{\int_{0}^{d} \sin \left[\phi_{2}(z)+x_{2}\right] \cos \left[\phi_{2}(z)+x_{2}\right] d z},
$$

for IPS-STN.

Using Eqs. (4a), (4b), (6a), (6b), (8a), and (8b), we can calculate the response time for the IPS-TN and IPS-STN cells. However, if the twist angle in Fig. 1(a) is between $90^{\circ}$ and $180^{\circ}$, we should treat it as two cascaded TN cells with the virtual division line parallel to the electric field direction so that we can still calculate its response time. The first TN cell has an effective twist angle of $90^{\circ}$ and an effective cell gap $d_{\text {eff, } 1}=d \pi / 2 \Phi$, while the second TN cell has an effective twist angle of $\Phi-90^{\circ}$ and an effective cell gap $d_{\text {eff }, 2}=d(1$ $-\pi / 2 \Phi)$. For the case of $\pi / 2<\Phi<\pi$, we find $d_{\text {eff, } 1}>d / 2$ and $d_{\text {eff,2 }}<d / 2$, which means that the first TN cell has a larger effective cell gap than the second TN cell. Since the response time is proportional to the square of cell gap, under such a circumstance, the device response time is determined by the slowest one, which is the first cell because $d_{\text {eff,1 }}$ $>d_{\text {eff,2. }}$. As a result, its response time can still be calculated by using Eqs. (4a), (6a), and (8a) as long as we substitute $d_{\mathrm{eff}, 1}=d \pi / 2 \Phi$ for $d$ because the twist angle in Fig. 1(a) is between $90^{\circ}$ and $180^{\circ}$. In our calculation, the following LC parameters are used: $K_{22}=7 \mathrm{pN}, \Delta \varepsilon=7.8, d=3 \mu \mathrm{m}$, and $\gamma_{1}$ $=0.1 \mathrm{~Pa} \mathrm{~s}$.

To ensure that the small angle approximation holds, let us assume that the maximum twist angle deformation is $0.05 \mathrm{rad}$ from the initial state. The required driving electric field strength [I for Fig. 1(a) and II for Fig. 1(b)] for different twist angles is plotted in Fig. 2. The corresponding rise time and relaxation time for these two configurations are plotted in Figs. 3(a) and 3(b), respectively. From Fig. 3(a), we find that the rise time decreases as the total twist angle increases for the IPS-TN cell, but increases for the IPS-STN cell. More importantly, IPS-STN has a much shorter rise time than IPSTN. The reasons are twofold. First, in order to have the same maximum twist angle deformation, IPS-STN cell needs a higher driving voltage than IPS-TN, as shown in Fig. 2. A larger driving electric field leads to a larger electric torque to the liquid crystal layer which makes the LC directors move faster. Second, in the IPS-STN cell, the middle layer LC directors are not reoriented by the external electric field; therefore, we can assume that an invisible boundary layer exists in the middle. Consequently, the equivalent cell gap is only one-half of the original one, resulting in a much faster dynamic response. From Fig. 3(b), we find that the relaxation time remains a constant $(\sim 3.25 \mathrm{~ms})$ for the IPS-STN cell, as indicated in Eq. (4b). For the IPS-TN cell, as we can see from curve I in Fig. 3(b), the relaxation time remains unchanged as the twist angle increases from $0^{\circ}$ to $90^{\circ}$ and then quickly decreases as the twist angle further increases from $90^{\circ}$ to $180^{\circ}$ because of the variety of the effective cell gap, which is discussed above. When the twist angle equals to $180^{\circ}$, the effective cell gap $d_{\text {eff, } 1}=d / 2$, which is identical to the IPS-STN cell shown in Fig. 1(b), the same response time can be seen from Fig. 3. Overall speaking, the device configuration shown in Fig. 1(b) exhibits a faster response time than that in Fig. 1(a) when the total twist angle is less than $180^{\circ}$.

In conclusion, we have analyzed the effect of twist angle on the response time of in-plane-switching mode LCDs. The response time depends strongly on the twist angle and the initial director configuration. Both rise time and relaxation time of IPS-STN configuration are faster than those of IPS-TN configuration due to the unactivated middle LC layer in the IPS-STN configuration. Our analysis results will help to improve the dynamic response performance of IPS mode LCDs.

This research was supported by the Key Construction Project of Hebei Provincial University and Natural Science Foundation of Hebei Province (Nos. A2006000675 and 103002), People's Republic of China.

${ }^{1}$ R. A. Soref, Appl. Phys. Lett. 22, 165 (1973).

${ }^{2}$ R. Kiefer, B. Weber, F. Windscheid, and G. Baur, Proceedings of the 12th International Display Research Conference, Hiroshima, Japan, 1992, p. 547.

${ }^{3}$ M. Oh-e and K. Kondo, Appl. Phys. Lett. 69, 623 (1996).

${ }^{4}$ M. Oh-e and K. Kondo, Liq. Cryst. 22, 379 (1997).

${ }^{5}$ Y. Sun, Z. Zhang, H. Ma, X. Zhu, and S. T. Wu, Appl. Phys. Lett. 81, 4907 (2002).

${ }^{6}$ Y. Sun and S. T. Wu, Jpn. J. Appl. Phys., Part 2 42, L423 (2003).

${ }^{7}$ S. H. Lee, J. G. You, H. Y. Kim, D. S. Lee, S. K. Kwon, H. S. Park, and C. K. Kim, SID Int. Symp. Digest Tech. Papers 28, 711 (1997).

${ }^{8}$ S. Oka, K. Kobayashi, Y. Iwamoto, Y. Toko, M. Kimura, and T. Akahane, Jpn. J. Appl. Phys., Part 1 42, 7445 (2003).

${ }^{9}$ S. Oka, K. Kobayashi, Y. Iwamoto, Y. Toko, M. Kimura, and T. Akahane, Jpn. J. Appl. Phys., Part 1 43, 3443 (2004).

${ }^{10}$ Y. Sun, H. Ma, Z. Zhang, and S. T. Wu, J. Appl. Phys. 92, 1956 (2002).

${ }^{11}$ M. Oh-e, M. Yoneya, and K. Kondo, J. Appl. Phys. 82, 528 (1997).

${ }^{12}$ P. Pieranski, F. Brockard, and E. Guyon, J. Phys. (Paris) 34, 35 (1975). 